\title{
Generalized Knaster-Kuratowski-Mazurkiewicz type theorems and applications to minimax inequalities
}

\author{
Ha Manh Linh
}

\begin{abstract}
Knaster-Kuratowski-Mazurkiewicz type theorems play an important role in nonlinear analysis, optimization, and applied mathematics. Since the first well-known result, many international efforts have been made to develop sufficient conditions for the existence of points intersection (and their applications) in increasingly general settings: Gconvex spaces [21, 23], L-convex spaces [12], and FCspaces $[8,9]$.
\end{abstract}

Applications of Knaster-Kuratowski-Mazurkiewicz type theorems, especially in existence studies for variational inequalities, equilibrium problems and more general settings have been obtained by many authors, see e.g. recent papers $[1,2,3,8,18,24,26]$ and the references therein.

In this paper we propose a definition of generalized KnasterKuratowski-Mazurkiewicz mappings to encompass R-KKM mappings [5], L-KKM mappings [11], T-KKM mappings [18, 19], and many recent existing mappings. Knaster-KuratowskiMazurkiewicz type theorems are established in general topological spaces to generalize known results. As applications, we develop in detail general types of minimax theorems. Our results are shown to improve or include as special cases several recent ones in the literature..

Index Terms - $L-T$-KKM mappings, Generalized convexity, Transfer compact semicontinuity, Minimax theorems, Saddle-points.

\section{INTRODUCTION}

$\mathrm{E}^{\mathrm{x}}$ xistence of solutions takes a central place in the optimization theory. Studies of the existence of solutions of a problem are based on existence results for important points in nonlinear analysis like fixed points, maximal points, intersection points, etc.

Manuscript Received on July 13 ${ }^{\text {th }}$, 2016. Manuscript Revised December 06 ${ }^{\text {th }}, 2016$.

This work was supported by University of Information Technology, Vietnam National University Hochiminh City under grant number D1-2017-07.

Ha Manh Linh was with the Department of Mathematics, Vietnam National University-HoChiMinh City, University of Information Technology, Thu Duc district, Saigon, Vietnam email:linhhm@uit.edu.vn.
One of the most famous existence theorems in nonlinear analysis is the classical KKM theorem, which has been generalized by many authors. For example see $[1,2,3,4,6,10,22,23,27]$. In early forms of this fundamental result, convexity assumptions played a crucial role and restricted the ranges of applicable areas. After, various generalized linear/convex structures have been proposed and corresponding types of KKM mappings have been defined together with these spaces, such as $[3,6,21]$ investigated G-convex spaces, Ding [7-9] introduced the concept of a FCspace and then Khanh and Quan [18, 19], Khanh, Lin and Long [14], Khanh and Long [15, 16] and, Khanh, Long and Quan [17] generalized and unified the previous spaces into a notion called a GFC-space.

Applications of KKM-type theorems, especially in existence studies for variational inequalities, equilibrium problems and more general settings have been obtained by many authors, see e.g. recent papers $[1,2,3,8,18,24,26]$ and the references therein.

To avoid in a stronger sense convexity structures in investigating KKM-type theorems, in this paper we propose a definition of a generalized type of KKM mappings in terms of a FLS-space and use it to establish generalized KKM type theorems. As applications we focus only on minimax and saddlepoint problems, which also generalize or improve recent results in the literature $[3,5,6,10, \ldots]$.

The outline of the paper is as follows. Section 2 contains definitions and preliminary facts for our later use. In Section 3, we give our main results. This section contains generalized KKM-type theorems, a Ky Fan type matching theorem and discuss their consequences in some particular cases. In section 4, we obtain the sufficient conditions for the solutions existence of minimax and saddlepoint problems. 


\section{PRELIMINARIES}

We recall now some definitions for our later use. For a set $X$, by $2^{X}$ and $\langle X\rangle$ we denote the family of all nonempty subsets, and the family of nonempty finite subsets, respectively. Let $Z, X$ be topological spaces and $A, B \subset Z, \operatorname{int} A, \operatorname{cl} A$ (or $\bar{A}$ ), int ${ }_{B} A$ and $\mathrm{cl}_{B} A$ stand for the interior, closure, interior in $B$ and closure in $B$ of $A, A$ is called compactly open (compactly closed, resp.) if for each nonempty compact subset $K$ of $Z, A \cap K$ is open (closed, resp.) in $K$. The compact interior and compact closure of $A$ are defined by

$$
\begin{aligned}
& \operatorname{cint} A=\bigcup\{B \subseteq Z: B \subseteq \text { AandBiscompactlyopenin } Z, \\
& \operatorname{ccl} A=\bigcap\{B \subseteq Z: B \supseteq \text { AandBiscompactlyclosedin } Z\} .
\end{aligned}
$$

It is clear that $\operatorname{cint} A$ ( $\operatorname{ccl} A$, resp.) is compactly open (compactly closed, resp.) in $Z$ and for each nonempty compact subset $K \subseteq Z$ with $A \cap K \neq \varnothing$, one has $K \cap \operatorname{cint} A=\operatorname{int}_{K}(K \cap A)$ and $K \cap \operatorname{ccl} A=\operatorname{cl}_{K}(K \cap A)$. It is equally obvious that $A \subseteq Z$ is compactly open (compactly closed, resp.) if and only if $\operatorname{cint} A=A(\operatorname{ccl} A=A$, resp.). A setvalued $T: X \rightarrow 2^{Z}$ is said to be upper [lower resp.] semicontinuous (usc) [lsc resp.] if for any open [closed resp.] subset $U \subset Z$, the set $T^{+}:=\{x \in X: T(x) \subset U\}$ is open [closed resp] in $X . T$ is said compact if $\overline{T(X)}$ is compact subset of $Z$.

$\mathrm{N}, \mathrm{Q}$, and $\mathrm{R}$ denote the set of the natural numbers, the set of rational numbers, and that of the real numbers, respectively, and $\bar{R}=\mathrm{R} \bigcup\{-\infty,+\infty\}$. For $n \in \mathrm{N}, \Delta_{n}$ stands for the $n$-simplex with the vertices being the unit vectors $e_{1}, e_{2}, \ldots, e_{n+1}$ of a basis of $\mathrm{R}^{n+1}$.

Definition 1 Let $X$ be a topological space, $Y$ be a nonempty set and $\Psi$ be a family of lower semicontinuous mappings $\psi: \Delta_{n} \rightarrow 2^{X}, n \in \mathrm{N}$. Then a triple $(X, Y, \Psi)$ is said to be a finitely lower semicontinuous topological space (FLS-space in short) if for each finite subset $N=\left\{y_{0}, y_{1}, \ldots, y_{n}\right\} \in\langle Y\rangle$, there is $\psi_{N}: \Delta_{n} \rightarrow 2^{X}$ of the family $\Psi$. Later we also use $\left(X, Y,\left\{\psi_{N}\right\}\right)$ to denote $(X, Y, \Psi)$.

Remark 1 If $\psi_{N}$ is a continuous single-valued mapping, then $(X, Y, \Psi)$ becomes an GFC-space as defitioned in [18-20]. If in addition $Y=X$ then $(X, Y, \Psi)$ is rewritten as $(X, \Psi)$ and becomes an $F C$-space in [7, 8]. The Example 1 below shows that in general the inverse is not true.
Definition 2 (See [18-20]). Let $(X, Y, \Phi)$ be a $G F C$-space and $Z$ be a topological space. Let $T: X \rightarrow 2^{Z}, F: Y \rightarrow 2^{Z}$ be two set-valued mappings. $F$ is called a generalized KKM mapping with respect to $T$ ( $T$-KKM mapping in short) if for each $N=\left\{y_{0}, \ldots, y_{n}\right\} \in\langle Y\rangle$ and each $\left\{y_{i_{0}}, \ldots, y_{i_{k}}\right\} \in\langle N\rangle$,

$$
T\left(\varphi_{N}\left(\Delta_{k}\right)\right) \subset \bigcup_{j=0}^{k} F\left(y_{i j}\right),
$$

where $\varphi_{N} \in \Phi$ is corresponding to $N$ and $\Delta_{k}=\operatorname{co}\left\{e_{i_{0}}, \ldots, e_{i_{k}}\right\}$.

Definition 3 (See [19]). Let (X,Y,Ф) be a GFCspace and $Z$ be a topological space. A set-valued mapping $T: X \rightarrow 2^{Z}$ is called better admissible if $T$ is usc and compact-valued such that for each $N \in\langle Y\rangle$ and each continuous mapping $\zeta: T\left(\varphi_{N}\left(\Delta_{n}\right)\right) \rightarrow \Delta_{n}$, the composition $\left.\zeta \circ T\right|_{\varphi_{N}\left(\Delta_{n}\right)}{ }^{\circ} \varphi_{N}: \Delta_{n} \rightarrow 2^{\Delta_{n}}$ has a fixed point, where $\varphi_{N} \in \Phi$ is corresponding to $N$.

The class of all such better admissible mapping from $X$ to $Z$ is denoted by $\mathrm{B}(X, Y, Z)$

Definition 4 (See [7]). Let $Z$ be a topological space and $Y$ be a nonempty set. Let $F: Y \rightarrow 2^{Z}$ is a set-valued mapping.

1. $F$ is called transfer open-valued (transfer closed-valued, resp.) if, for each $y \in Y$ and $z \in F(y)$ $\left(z \notin F(y)\right.$, resp.) there exists $y^{\prime} \in Y$ such that $z \in \operatorname{int} F\left(y^{\prime}\right) \quad\left(z \notin \mathrm{cl} F\left(y^{\prime}\right)\right.$, resp. $)$

2. $F$ is said to be transfer compactly openvalued (transfer compactly closed-valued, resp.) if for each $y \in Y$, each nonempty compact subset $K \subseteq Z$ and each $z \in F(y) \cap K \quad(z \notin F(y) \cap K$, resp. $)$, there is $y^{\prime} \in Y \quad$ such that $z \in$ int $_{K}\left(F\left(y^{\prime}\right) \cap K\right)$ $\left(z \notin \mathrm{cl}_{K}\left(F\left(y^{\prime}\right) \cap K\right)\right.$, resp.)

We will need the following well-known result.

Lemma 1 ([7]). Let $Y$ be a set, $X$ be a topological space and $F: Y \rightarrow 2^{X}$. The following statements are equivalent.

1. $\mathrm{F}$ is transfer compactly closed-valued (transfer compactly open-valued, respectively).

2. for each compact subset $K \subset X$. 


$$
\begin{aligned}
\bigcap_{y \in Y}(F(y) \cap K) & =\bigcap_{y \in Y}(\operatorname{ccl} F(y) \cap K) \\
& =\bigcap_{y \in Y}\left(\mathrm{cl}_{K} F(y) \cap K\right), \\
\bigcup_{y \in Y}(F(y) \cap K) & =\bigcup_{y \in Y}(\operatorname{cint} F(y) \cap K) \\
& =\bigcup_{y \in Y}\left(\operatorname{int}_{K} F(y) \cap K\right) .
\end{aligned}
$$

Definition 5 Let $(X, Y, \Psi)$ be a FLS-space and $Z$ be a topological space. Let $F: Y \rightarrow 2^{Z}$ and $T: X \rightarrow 2^{Z}$ be set-valued mappings

1. $F$ is said to be a generalized $L-\mathrm{KKM}$ mapping wrt $T$ ( $L-T$-KKM mapping in short) if, for each $N=\left\{y_{0}, y_{1}, \ldots, y_{n}\right\} \in\langle Y\rangle \quad$ and each $\left\{y_{i_{0}}, y_{i_{1}}, \ldots, y_{i_{k}}\right\} \subseteq N$, one has $T\left(\psi_{N}\left(\Delta_{k}\right)\right) \subseteq \bigcup_{j=0}^{k} F\left(y_{i_{j}}\right)$, where $\psi_{N} \in \Psi$ is corresponding to $N$ and $\Delta_{k}=\operatorname{co}\left\{e_{i_{0}}, e_{i_{1}}, \ldots, e_{i_{k}}\right\}$.

2. We say that a set-valued mapping $T: X \rightarrow 2^{Z}$ has the generalized $L-\mathrm{KKM}$ property if, for each $L-T-\mathrm{KKM}$ mapping $F: Y \rightarrow 2^{Z}$, the family $\{\overline{F(y)}: y \in Y\}$ has the finite intersection property, i.e. all finite intersections of sets of this family are nonempty. The class of all mappings $T: X \rightarrow 2^{Z}$ which have the generalized $L-\mathrm{KKM}$ property is denoted by $L-\operatorname{KKM}(\mathrm{X}, \mathrm{Y}, \mathrm{Z})$.

3. Let $S: Y \rightarrow 2^{X}$ be a set-valued mapping. A subset $D$ of $Y$ is called an $L-S$-subset of $Y$ if, for each $N=\left\{y_{0}, \ldots, y_{n}\right\} \in\langle Y\rangle$ and each $\left\{y_{i_{0}}, \ldots, y_{i_{k}}\right\} \subseteq N \cap D$, one has $\psi_{N}: \Delta_{n} \rightarrow 2^{X}$ of $\Psi$ such that $\psi_{N}\left(\Delta_{k}\right) \subseteq S(D)$, where $\Delta_{k}$ is face of $\Delta_{n}$ corresponding to $\left\{y_{i_{0}}, \ldots, y_{i_{k}}\right\}$.

Remark 2 Note that the Definition 5 (i) is a generalization of the Definition 2.1 of [11]. We also see that every $L-T-K K M$ mapping is a $T$ KKM mapping when $\psi_{N}$ is a continuous singlevalued mapping. If in addition $Y=X$ and $T$ is the identity map then $L-T-K K M$ mapping becomes an $R-K K M$ mapping of [5] and thw Definition 2.2 of [7].

The following example shows that the Definition 5 (i) contains the Definition 2.
Example 1 Suppose that $X=Z=[0,+\infty)$ and $Y=\mathrm{N}$. For each $N \in\langle Y\rangle$, we define $\varphi_{N}: \Delta_{n} \rightarrow 2^{X}$ by

$$
\Psi_{N}(e)= \begin{cases}\{0\}, & \text { if } e \in\left\{e_{0}, \ldots, e_{n}\right\}, \\ , & \text { otherwise. }\end{cases}
$$

We see that $\psi_{N}$ is lower semicontinuous but not continuous. Hence $(X, Y, \Psi)$ is a FLS-space.

Let $F: Y \rightarrow 2^{Z}$ and $T: X \rightarrow 2^{Z}$ be defined as follows $F(y)=[0, y+2)$ for each $y \in Y$ and $T(x)=[0,1]$ foreach $x \in X$. Then $F$ is not a $T-\mathrm{KKM}$ mapping. However $F$ is the generalized $L-T$ KKM mapping. Also, the class $\{\overline{F(y}: y \in Y\}$ has the finite intersection property.

Lemma 2 (Classical) Let $T: X \rightarrow 2^{Z}$ be upper semicontinuous with compact valued from $a$ compact space $X$ to $Y$. Then $T(X)$ is compact.

Lemma 3 Let $(X, Y, \Phi)$ be a GFC-space and $Z$ be a topological space. Then $\mathrm{B}(X, Y, Z) \subseteq L$ $\operatorname{KKM}(X, Y, Z)$.

Proof. For each $T \in \mathrm{B}(X, Y, Z)$, let $F$ is a generalized $L-T-\mathrm{KKM}$. Suppose to the contrary that $N=\left\{y_{0}, \ldots, y_{n}\right\} \in\langle Y\rangle$ exists such that

$$
\bigcap_{i=0}^{n} \overline{F\left(y_{i}\right)}=\varnothing \text {. }
$$

It follows that

$$
\overline{T\left(\varphi_{N}\left(\Delta_{n}\right)\right)} \cap \bigcap_{i=0}^{n} \overline{F\left(y_{i}\right)}=\varnothing
$$

and

$$
\overline{T\left(\varphi_{N}\left(\Delta_{n}\right)\right)}=\bigcup_{i=0}^{n}\left[\left(Z \backslash \overline{F\left(y_{i}\right)}\right) \cap \overline{T\left(\varphi_{N}\left(\Delta_{n}\right)\right)}\right] .
$$

Then $\left\{\left(Z \backslash \overline{F\left(y_{i}\right)}\right) \cap \overline{T\left(\varphi_{N}\left(\Delta_{n}\right)\right)_{i=0}^{n}} \quad\right.$ is an open covering of the compact set $\overline{T\left(\varphi_{N}\left(\Delta_{n}\right)\right)}$. Let $\left\{\zeta_{i}\right\}_{i=0}^{n}$ be a continuous partition of unit associated with this covering and $\zeta: \overline{T\left(\varphi_{N}\left(\Delta_{n}\right)\right)} \rightarrow \Delta_{n}$ be defined by $\zeta(t)=\sum_{i=0}^{n} \zeta_{i}(t) e_{i}$. Then $\zeta$ is continuous. Since $T \in \mathrm{B}(X, Y, Z)$, the composition $\left.\zeta \circ T\right|_{\varphi_{N}\left(\Delta_{n}\right)}{ }^{\circ} \varphi_{N}$ has a fixed point. Hence, there is $z_{0} \in T\left(\varphi_{N}\left(\Delta_{n}\right)\right)$ such that $z_{0} \in T\left(\varphi_{N}\left(\zeta\left(z_{0}\right)\right)\right)$. Where $\zeta\left(z_{0}\right)=\sum_{j \in J(0)} \zeta_{j}\left(z_{0}\right) e_{i} \in \Delta_{J\left(z_{0}\right)} \quad$ with $\left.J_{(} z_{0}\right)=\left\{j \in\{0,1, \ldots, n\}: \zeta_{j}\left(z_{0}\right) \neq 0\right\}$ 
On the other hand, as $F$ is $L-T-\operatorname{KKM}(T-$

KKM), one has

$$
\begin{aligned}
z_{0} \in \overline{T\left(\varphi_{N}\left(\zeta\left(z_{0}\right)\right)\right)} & \subseteq \overline{T\left(\varphi_{N}\left(\Delta_{J\left(z_{0}\right)}\right)\right)} \\
& \subseteq \overline{\bigcup_{j \in J\left(z_{0}\right)} F\left(y_{j}\right)} \\
& =\bigcup_{j \in J\left(z_{0}\right)} \overline{F\left(y_{j}\right)}
\end{aligned}
$$

so there is $\bar{j} \in J\left(z_{0}\right)$ such that $z_{0} \in \overline{F\left(y_{j}^{-}\right)}$

However, in view of the definitions of $J\left(z_{0}\right)$ and

$$
\begin{aligned}
\text { of } & \text { the } \quad \text { partition } \\
z_{0} & \left.\in\left\{z \in \overline{T\left(\varphi_{N}\left(\Delta_{n}\right)\right)}: \zeta_{\bar{j}}\right\}_{i=0}^{n}(z) \neq 0\right\} \\
& \subseteq\left(Z \backslash \overline{F\left(y_{\bar{j}}\right)}\right) \cap \overline{T\left(\varphi_{N}\left(\Delta_{n}\right)\right)} \\
& \subseteq Z \backslash \overline{F\left(y_{j}^{-}\right)},
\end{aligned}
$$

a contradiction.

\section{GENERALIZED $L-T$-KKM TYPE THEOREMS}

Theorem 1 Let $(X, Y, \Psi)$ be a FLS-space and $Z$ be topological spaces. Let $F: Y \rightarrow 2^{Z}$ and $T: X \rightarrow 2^{Z}$ be set-valued mappings. Assume that the following conditions hold

1. $\mathrm{F}$ is L-T-KKM;

2. $T \in \mathrm{L}-\mathrm{KKM}(\mathrm{X}, \mathrm{Y}, \mathrm{Z})$ and $\overline{T(X)}$ is a compact subset of $Z$;

3. there are $A \in\langle Y\rangle$ and a nonempty compact subset $\mathrm{K}$ of $\mathrm{Z}$ such that

$$
\bigcap_{y \in A} \operatorname{ccl} F(y) \subseteq K
$$

4. F is transfer compactly closed-valued. Then

$$
K \cap \overline{T(X)} \cap\left(\bigcap_{y \in Y} F(y)\right) \neq \varnothing .
$$

Proof. Define a new set-valued mapping $H: Y \rightarrow 2^{\overline{T(X)}}$ by

$H(y)=\overline{T(X)} \cap \operatorname{ccl} F(y)$, for each $y \in Y$.

Then $H$ has closed-values in $\overline{T(X)}$. We show that $H$ is $L-T-\mathrm{KKM}$. Indeed, since $F$ is $L-T$ $\mathrm{KKM}$, for each $N=\left\{y_{0}, \ldots, y_{n}\right\} \in\langle Y\rangle$ and each $\left\{y_{i_{0}}, \ldots, y_{i_{k}}\right\} \subseteq N$ one has

$$
\begin{aligned}
T\left(\psi_{N}\left(\Delta_{k}\right)\right) & =T\left(\psi_{N}\left(\Delta_{k}\right)\right) \cap \overline{T(X)} \\
& \subseteq \bigcup_{j=0}^{k} F\left(y_{i_{j}}\right) \cap \overline{T(X)} \\
& =\bigcup_{j=0}^{k}\left[F\left(y_{i_{j}}\right) \cap \overline{T(X)}\right] \\
& \subseteq \bigcup_{j=0}^{k} H\left(y_{i_{j}}\right) .
\end{aligned}
$$

Therefore, $H$ is the $L-T-\mathrm{KKM}$ mapping.

Moreover, since $T \in L-\mathrm{KKM}(\mathrm{X}, \mathrm{Y}, \mathrm{Z})$ it follows that the family

$$
\{\overline{H(y)}: y \in Y\}=\{H(y): y \in Y\}
$$

has the finite intersection property. Since $\overline{T(X)}$ is compact and $\{H(y): y \in Y\}$ is a family of closed subsets in $\overline{T(X)}$, one has

$\varnothing \neq \bigcap_{y \in Y} H(y)=\bigcap_{y \in Y}(\overline{T(X)} \cap \operatorname{ccl} F(y))$.

Hence, there exists $\hat{z} \in \bigcap_{y \in Y}(\overline{T(X)} \cap \operatorname{ccl} F(y))$, i.e., $\hat{z} \in \operatorname{ccl} F(y)$ ), for each $y \in Y$. By (iii), there is $A \in\langle Y\rangle$ and a compact subset $K$ of $Z$ such that

$$
\hat{z} \in \bigcap_{y \in A} \operatorname{ccl} F(y) \subseteq K .
$$

By (iv) and Lemma 1, we have

$$
\begin{aligned}
z & \in \bigcap_{z \in Y} \operatorname{ccl} F(z) \cap \overline{T(X)} \\
& =\bigcap_{z \in Y} F(z) \cap \overline{T(X)} \\
& \subset F(y) .
\end{aligned}
$$

Thus we arrive at the conclusion

$$
K \cap \overline{T(X)} \cap\left(\bigcap_{y \in Y} F(y)\right) \neq \varnothing .
$$

Remark 3 Theorem 1 unifies and generalizes Theorem 3.2 of [5], Theorem 3.2 of [11] and Theorem 3.2 of [21] under much weaker assumptions. By Lemma 3, Theorem 1 improves the assertion ( iii $_{1}$ ) of Theorem 2.2 of [19].

The following example shows that we cannot use of known results in $F C$-spaces of [7] or $G F C$ convex spaces of [18-20], but is easily investigated by $F L S$-spaces.

Example 2 Let $Y=\mathrm{N} \cup\{0\}$ and $X=Z=[0 ;+\infty)$. For each $N=\left\{y_{0}, y_{1}, \ldots, y_{n}\right\} \in\langle Y\rangle$, we define $\varphi_{N}: \Delta_{n} \rightarrow X$, by $\varphi_{N}(e)=\sum_{i=0}^{n} \lambda_{i} y_{i}$, where $e=\sum_{i=0}^{n} \lambda_{i} e_{i} \in \Delta_{n}$ and 
$\sum_{i=0}^{n} \lambda_{i}=1$. Then $\left(X, Y,\left\{\varphi_{N}\right\}\right)$ is the GFC-space. Let $F: Y \rightarrow 2^{Z}$ and $T: X \rightarrow 2^{Z}$ are defined as follows

$F(y)=\{\{0\}$ if $\mathrm{y}=0,[0,0.5]$ if otherwise.

$$
T(x)=\{\{0\} \quad[0,1),[0,1] \text { if otherwise.. }
$$

We can see that $F$ is not $T$-KKM. Indeed, we choose $N_{*}=\left\{y_{0}=1\right\} \in\langle Y\rangle$, one has

$$
\begin{aligned}
& \varphi_{N_{*}}\left(\Delta_{0}\right)=1 \text { and } \\
& T\left(\varphi_{N_{*}}\left(\Delta_{0}\right)\right)=[0,1] \cup ́[0,0.5]=F(1) .
\end{aligned}
$$

Hence the results in [18-20] are out of use for this case.

To apply our Theorem 1, we now define a $F L S$ space by $Y=\mathrm{N} \cup\{0\}, \quad X=[0 ;+\infty)$ and the corresponding $\psi_{N}: \Delta_{n} \rightarrow 2^{X}$, by

$$
\psi_{N}(e) \quad=\quad\{0\} \quad \text { if } \quad \mathrm{e} \in\left\{e_{0}, \ldots, e_{n}\right\},[0 ;
$$

0.5 ] if otherwise..

We see that $\psi_{N}$ is lower semicontinuous mapping, so $\left(X, Y\left\{\psi_{N}\right\}\right)$ is a $F L S$-space. Furthermore, for each $N=\left\{y_{0}, y_{1}, \ldots, y_{n}\right\} \in\langle Y\rangle$ we have

$T\left(\psi_{N}\left(\Delta_{n}\right)\right)=\{0\} \subseteq F(y)$ for each $y \in Y$

Therefore $F$ is a $L-T-\mathrm{KKM}$ mapping, so (i) of Theorem 1 is satisfied. Clearly $\overline{T(X)}=[0,1]$ is the compact subset of $Z$ and the class $\{\overline{F(y}: y \in Y\}$ has the finite intersection property,i.e., (ii)of Theorem 1 is fulfilled. If we choose $A=\{0,1\}$ and $K=[0,1]$ then assumptions (iii) of Theorem 1 are satisfied. Moreover it is easy to see that $F$ is transfer compactly closed-valued. By Theorem 1, one concludes that

$$
K \cap \overline{T(X)} \cap\left(\bigcap_{y \in Y} F(y)\right)=\{0\} \neq \varnothing .
$$

Theorem 2 Let $(X, Y, \Psi)$ be a FLS -space and $Z$ be topological spaces. Let $S: Y \rightarrow 2^{X}, \quad F: Y \rightarrow 2^{Z}$ and $T: X \rightarrow 2^{Z}$ be set-valued mappings. Assume that $Y$ is an L-S-subset of itself. Let the following conditions hold

1. $\mathrm{F}$ is L-T-KKM and transfer compactly closed-valued;

2. $T \in L-\mathrm{KKM}(\mathrm{X}, \mathrm{Y}, \mathrm{Z}), \overline{T(S(Y))}$ is a compact subset of $\mathrm{Z}$.

Then

$\overline{T(S(Y))} \cap \bigcap_{y \in Y} F(y) \neq \varnothing$.

Proof. We define a set-valued mapping $H: Y \rightarrow 2^{\overline{T(S(Y))}}$ by

$H(y)=\overline{T(S(Y))} \cap \operatorname{ccl} F(y)$, for each $y \in Y$.
Then $H$ has closed values in $\overline{T(S(Y))}$. We show that $H$ is $L-T-\mathrm{KKM}$. Indeed, by $F$ is $L-T-\mathrm{KKM}$ mapping, for any $N=\left\{y_{0}, \ldots, y_{n}\right\} \in\langle Y\rangle$, and any $\left\{y_{i_{0}}, \ldots, y_{i_{k}}\right\} \in\langle N\rangle$ one has $T\left(\psi_{N}\left(\Delta_{k}\right)\right) \subseteq \bigcup_{j=0}^{k} F\left(y_{i_{j}}\right)$. Since $Y$ is $L-S$-subset of $Y, T\left(\psi_{N}\left(\Delta_{k}\right)\right) \subseteq \overline{T(S(Y))}$.

Therefore

$$
\begin{aligned}
T\left(\psi_{N}\left(\Delta_{k}\right)\right) & =T\left(\psi_{N}\left(\Delta_{k}\right)\right) \cap \overline{T(S(Y))} \\
& \subseteq \bigcup_{j=0}^{k} F\left(y_{i_{j}}\right) \cap \overline{T(S(Y))} \\
& =\bigcup_{j=0}^{k}\left[F\left(y_{i_{j}}\right) \cap \overline{T(S(Y))}\right] \\
& \subseteq \bigcup_{j=0}^{k} H\left(y_{i_{j}}\right) .
\end{aligned}
$$

Hence, $\quad H$ is $L-T$-KKM. As $T \in L-$ $\operatorname{KKM}(X, Y, Z)$, it follows that the family $\{\overline{H(y)}: y \in Y\}=\{H(y): y \in Y\}$ has the finite intersection property. Since $\overline{T(S(Y))}$ is compact and $\{H(y): y \in Y\}$ is a family of closed subsets in $\overline{T(S(Y))}$ and by

$$
\begin{aligned}
\overline{T(S(Y))} \cap \bigcap_{y \in Y} F(y) & =\bigcap_{y \in Y}(\overline{T(S(Y))} \cap F(y)) \\
& =\bigcap_{y \in Y}(\overline{T(S(Y))} \cap \operatorname{ccl} F(y)) \\
& =\bigcap_{y \in Y} H(y) \neq \varnothing .
\end{aligned}
$$$$
\text { Lemma } 1 \text {, we have }
$$

Remark 4 Theorem 2 contains Theorem 1 of [21], Theorem 3.1, 3.2 and 3.3 of [7] and Theorem 3.1 of [18].

Theorem 3 Let $(X, Y, \Psi)$ be a FLS -space and $Z$ be topological spaces. Let $S: Y \rightarrow 2^{X}, G: Z \rightarrow 2^{Y}$ and $T: X \rightarrow 2^{Z}$ be set-valued mappings. Assume that $Y$ is an L-S-subset of itself. Let the following conditions hold

1. $G^{-1}$ is transfer compactly open-valued;

2. for each $N \in\langle Y\rangle$ and each $\left\{y_{i_{0}}, \ldots, y_{i_{k}}\right\} \subseteq N$,

$$
T\left(\psi_{N}\left(\Delta_{k}\right)\right) \cap \bigcap_{j=0}^{k} G^{-1}\left(y_{i_{j}}\right)=\varnothing
$$

3. $T \in L-\mathrm{KKM}(\mathrm{X}, \mathrm{Y}, \mathrm{Z}), \overline{T(S(Y))}$ is a compact subset of $Z$.

Then there exists $\hat{z} \in \overline{T(S(Y))}$ such that $G(\hat{z})=\varnothing$

Proof. To apply Theorem 2, we define a new set-valued mapping $F: Y \rightarrow 2^{Z}$ by

$$
F(y)=Z \backslash G^{-1}(y) \text {, for each } y \in Y \text {. }
$$


Then $F$ is transfer compactly closed-valued. We show that $F$ is $L-T-K K M$. Indeed, by (ii) for any $N=\left\{y_{0}, \ldots, y_{n}\right\} \in\langle Y\rangle$ and any $\left\{y_{i_{0}}, \ldots, y_{i_{k}}\right\} \in\langle N\rangle$, one has $T\left(\psi_{N}\left(\Delta_{k}\right)\right) \cap \bigcap_{j=0}^{k} G^{-1}\left(y_{i_{j}}\right)=\varnothing$, it follows that

$$
\begin{aligned}
T\left(\psi_{N}\left(\Delta_{k}\right)\right) & \subseteq Z \backslash \bigcap_{j=0}^{k} G^{-1}\left(y_{i_{j}}\right) \\
& =\bigcup_{j=0}^{k} F\left(y_{i_{j}}\right) .
\end{aligned}
$$

Therefore $F$ is a $L-T-\mathrm{KKM}$ mapping. It is clear to see that all conditions of Theorem 2 are satisfied. By Theorem 2

$$
\overline{T(S(Y))} \cap \bigcap_{y \in Y} F(y) \neq \varnothing .
$$

Hence, there exists

$$
\hat{z} \in \overline{T(S(Y))} \cap \bigcap_{y \in Y} F(y) .
$$

It follows that

$$
\hat{z} \in Z \backslash G^{-1}(y) \text { for each } y \in Y \text {, }
$$

i.e, $\hat{z} \notin G^{-1}(y)$ for each $y \in Y$. W

Thus, there exists $\hat{z} \in \overline{T(S(Y))}$ such that $G(\hat{z})=\varnothing$.

Remark 5 Theorem 3 contains the assertion (iii $\left.{ }_{1}\right)$ of Theorem 4.1 of [19] and Theorem 3.1 of [8].

As a consequence of the generalized $L-T-\mathrm{KKM}$ theorems, we prove a generalization of the Ky fan type matching theorem.

Theorem 4 Let $(X, Y, \Psi)$ be a FLS -space and $Z$ be a topological space. Let $S: Y \rightarrow 2^{X}, F: Y \rightarrow 2^{Z}$ and $T: X \rightarrow 2^{Z}$ be set-valued mappings. Assume that $Y$ is an L-S-subset of itself. Let the following conditions hold.

1. $\mathrm{F}$ is a transfer compactly open-valued mapping;

2. $T \in \mathrm{L}-\mathrm{KKM}(\mathrm{X}, \mathrm{Y}, \mathrm{Z})$, and $\overline{T(S(Y))}$ is compact;

3. $\overline{T(S(Y))} \subset F(Y)$.

Then, there exist $M=\left\{y_{0}, \ldots, y_{m}\right\} \in\langle Y\rangle$ and $\left\{y_{i_{0}}, \ldots, y_{i_{k}}\right\} \subseteq M$ such that

$$
T\left(\psi_{M}\left(\Delta_{k}\right)\right) \cap \bigcap_{j=0}^{k} F\left(y_{i_{j}}\right) \neq \varnothing .
$$

Proof. Suppose that the conclusion is not true. Then for any $N=\left\{y_{0}, \ldots, y_{n}\right\} \in\langle Y\rangle$ and any $\left\{y_{i_{0}}, \ldots, y_{i_{k}}\right\} \subseteq N, \quad T\left(\psi_{N}\left(\Delta_{k}\right)\right) \cap \bigcap_{j=0}^{k} F\left(y_{j}\right)=\varnothing$.

Therefore $\quad T\left(\psi_{N}\left(\Delta_{k}\right)\right) \subseteq \bigcup_{j=0}^{k} H\left(y_{j}\right), \quad$ where $H(y)=Z \backslash F(y)$. It follows that $H$ is $L-T$-KKM. By (i), $H$ is transfer compactly closed-valued. Clearly, all conditions of Theorem 2 are satisfied. It follows from Theorem 2 that

$$
\overline{T(S(Y))} \cap \bigcap_{y \in Y} H(y) \neq \varnothing .
$$

Hence, $\overline{T(S(Y))} \ddot{O} F(Y)$, but this contradictions (iii). Thus there exist $M=\left\{y_{0}, \ldots, y_{m}\right\} \in\langle Y\rangle$ and $\left\{y_{i_{0}}, \ldots, y_{i_{k}}\right\} \subseteq M$ such that

$$
T\left(\psi_{M}\left(\Delta_{k}\right)\right) \cap \bigcap_{j=0}^{k} F\left(y_{j}\right) \neq \varnothing .
$$

Remark 6 Theorem 3 generalizes Theorem 8 of [21] and Theorem 3.1 of [12] since being $G-K K M$ mapping and $R-K K M$ mapping are special cases of $L-T-K K M$ mapping.

Theorem 5 Theorem 2 and 4 are equivalent.

Proof. We saw that Theorem 4 can be proved by using Theorem 2. Now we derive Theorem 2 from Theorem 4. Suppose that

$$
\overline{T(S(Y))} \cap \bigcap_{y \in Y} F(y)=\varnothing .
$$

Let $H(y)=Z \backslash F(y)$. Then $H(y)$ is transfer compactly open-valued and $\overline{T(S(Y))} \subset H(Y)$. It follows from Theorem 4 that there exist $M \in\langle Y\rangle$ and $\quad\left\{y_{i_{0}}, \ldots, y_{i_{k}}\right\} \subseteq M \quad$ such that $T\left(\psi_{M}\left(\Delta_{k}\right)\right) \cap \bigcap_{j=0}^{k} H\left(y_{i_{j}}\right) \neq \varnothing, \quad$ (where $\left.\quad \psi_{M} \in \Psi\right)$. Hence $T\left(\varphi_{M}\left(\Delta_{k}\right)\right) \ddot{\theta} \bigcup_{j=0}^{k} F\left(y_{i_{j}}\right)$. This contradicts the fact that $F$ is $L-T-\mathrm{KKM}$. Thus the conclusion of Theorem 2 follows Theorem 4 .

\section{KY FAN TYPE MINIMAX INEQUALITIES}

In this section, by applying $L-T-\mathrm{KKM}$ theorems, we shall establish some new Ky Fan type minimax inequalities and saddle point problems.

Definition 6 Let $(X, Y, \Psi)$ be a $F L S$-space and $Z$ be a topological space. Let $T: X \rightarrow 2^{Z}, g: Y \times Z \rightarrow \mathrm{R} \cup\{ \pm \infty\}$ and $\lambda \in \mathrm{R} . g$ is called $\lambda-L$-quasiconvex ( $\lambda-L$-quasiconcave, resp.) wrt $T \quad$ in $y$ if, $\forall N \in\langle Y\rangle$ and 
$\forall\left\{y_{i_{0}}, \ldots, y_{i_{k}}\right\} \subseteq N, \forall z \in T\left(\psi_{N}\left(\Delta_{k}\right)\right), \quad$ one has $\max _{0 \leq j \leq k} g\left(y_{i}, z\right) \geq \lambda\left(\min _{0 \leq j \leq k} g\left(y_{i j}, z\right) \leq \lambda\right.$, resp. $)$.

Remark 7 Definition 6 generalizes Definition 4.1 of [9], Definition 4.1 of [20] and Definition 1.7 of [25]

Definition 7 Let $(X, Y, \Psi)$ be a $F L S$-space and $Z$ be a topological space. Let $T: X \rightarrow 2^{Z}, g: Y \times Z \rightarrow \overline{\mathrm{R}}$ and $\alpha, \beta \in \mathrm{R}$ with $\alpha \leq \beta . g$ is called $\alpha-\beta-L$-quasiconcave wrt $T$ in $y$ if, $\forall N \in\langle Y\rangle, \forall\left\{y_{i_{0}}, \ldots, y_{i_{k}}\right\} \subseteq N, \forall z \in T\left(\psi_{N}\left(\Delta_{k}\right)\right)$, there is an $r \in\{0, \ldots, k\}$ satisfying $\alpha \leq g\left(y_{i_{r}}, z\right) \leq \beta$. If $\alpha=-\infty$, then the notion in Definition 7 reduces to the corresponding notion in Definition 6.

We need also the following notion of Definition 2.6 in [6].

Definition 8 Let $Y$ be a nonempty set and $Z$ be a topological space. Let $f: Y \times Z \rightarrow \overline{\mathrm{R}}$ and $\lambda \in \mathrm{R} . f$ is called $\lambda$-transfer compactly lower (upper, resp.) semicontinuous in $z$ if for each compact subset $K$ of $Z$ and for each $z \in K$, there exists $a \quad y \in Y$ such that $f(y, z)>\lambda \quad(f(y, z)<\lambda$, resp., $)$ implies that there exists an open neighborhood $U(z)$ of $z$ and a point $y_{0} \in Y$ such that $f\left(y_{0}, z^{\prime}\right)>\lambda\left(f\left(y_{0}, z^{\prime}\right)<\lambda\right.$, resp., $)$ for all $z^{\prime} \in U(z)$.

Let $\quad F: Y \rightarrow 2^{Z} \quad$ by $\quad F(y)=\{z \in Z: f(y, z) \leq \lambda\}$ $(f(y, z) \geq \lambda$, resp.). Then $f$ is $\lambda$-transfer compactly lower (upper, resp.) semicontinuous in $z$ if and only if $F$ is transfer compactly closed-valued (open-valued resp.).

Theorem 6 Let $(X, Y, \Psi)$ be a FLS-space and $Z$ be a topological space. Let $T: X \rightarrow 2^{Z}, f, g: Y \times Z \rightarrow \mathrm{R} \cup\{ \pm \infty\}$ and $\lambda \in \mathrm{R}$ be such that

1. for each $(y, z) \in Y \times Z, f(y, z) \leq g(y, z)$;

2. $\mathrm{g}$ is generalized $\lambda$-L-quasiconcave wrt $\mathrm{T}$ in $\mathrm{y}$;

3. $\mathrm{f}$ is $\lambda$-transfer compactly in $\mathrm{z}$;

4. $\mathrm{T} \in \mathrm{L}-\mathrm{KKM}(\mathrm{X}, \mathrm{Y}, \mathrm{Z})$ and $\overline{T(X)}$ is a compact subset of $\mathrm{Z}$;

5. there exist $A \in\langle Y\rangle$ and a nonempty compact subset $\mathrm{K}$ of $\mathrm{Z}$ such that the set $\bigcap_{y \in A} \operatorname{ccl}\{z \in Z: f(y, z) \leq \lambda\} \subseteq K$.

Then there exists a point $\hat{z} \in Z$ such that $f(y, \hat{z}) \leq \lambda, \forall y \in Y$.
Proof. First, we define two set-valued mappings $F, G: Y \rightarrow 2^{Z}$ by

$F(y)=\{z \in Z: f(y, z) \leq \lambda\}$

and $G(y)=\{z \in Z: g(y, z) \leq \lambda\}, \forall y \in Y$.

By (i), we have that $G(y) \subseteq F(y), \forall y \in Y$. By (ii) and Definition 6, for each $N=\left\{y_{0}, \ldots, y_{n}\right\} \in\langle Y\rangle$, each $\left\{y_{i_{0}}, \ldots, y_{i_{n}}\right\} \in\langle N\rangle \quad$ and each $\quad z \in T\left(\psi_{N}\left(\Delta_{k}\right)\right)$, $\min _{0 \leq j \leq k} g\left(y_{i_{j}}, z\right) \leq \lambda$. Hence there exists $r \in\{0, \ldots, k\}$ such that $g\left(y_{i_{r}, z}\right) \leq \lambda, \quad$ i.e., $z \in G\left(y_{i_{r}}\right) \subset \bigcup_{j=0}^{k} G\left(y_{i}\right) \subset \bigcup_{j=0}^{k} F\left(y_{i_{j}}\right) . \quad$ Since $z \in T\left(\psi_{N}\left(\Delta_{k}\right)\right)$ is arbitrary, we have

$$
T\left(\psi_{N}\left(\Delta_{k}\right) \subset \bigcup_{j=0}^{k} F\left(y_{i j}\right) .\right.
$$

Hence, $F$ is a generalized $L-T-$ KKM mapping. The condition (iii) implies that $F$ is transfer compactly closed-valued. The condition (v) implies that there exists $A \in\langle Y\rangle$ and a nonempty compact subset $K$ of $Z$ such that

$\cap y \in A$ c $c l F(y) \subset K$.

Add the condition (iv), all conditions of Theorem 1 are satisfied. By Theorem 1 we have,

$$
\cap_{y \in Y} F(y) \neq \varnothing \text {. }
$$

Taking any $\hat{z} \in \cap_{y \in Y} F(y)$, we obtain $f(y, \hat{z}) \leq \lambda, \forall y \in Y$. W

Remark 8 Theorem 6 generalize Theorem 2.12.4 of [26].

Theorem 7 Let $(X, Y, \Psi)$ be a FLS-space and Z be a topological space. Let $T: X \rightarrow 2^{Z}, f, g: Y \times Z \rightarrow \mathrm{R} \cup\{ \pm \infty\}$ and $\lambda \in \mathrm{R}$ be such that

1. for each $(y, z) \in Y \times Z, f(y, z) \leq g(y, z)$;

2. $\mathrm{g}$ is generalized $\lambda$-L-quasiconcave wrt $\mathrm{T}$ in $\mathrm{y}$;

3. $\mathrm{f}$ is $\lambda$-transfer compactly lower semicontinuous in $\mathrm{z}$;

4. $T \in \mathrm{L}-\mathrm{KKM}(\mathrm{X}, \mathrm{Y}, \mathrm{Z})$; There is $S: Y \rightarrow 2^{X}$ such that $\mathrm{Y}$ is an L-S-subset of itself and $\overline{T(S(Y))}$ is compact.

Then there exists a point $\hat{z} \in Z$ such that $f(y, \hat{z}) \leq \lambda, \forall y \in Y$.

Proof. Define two set-valued mappings $F, G: Y \rightarrow 2^{Z}$ by

$F(y)=\{z \in Z: f(y, z) \leq \lambda\}$ and

$G(y)=\{z \in Z: g(y, z) \leq \lambda\}, \forall y \in Y$

By (i), we have that $G(y) \subseteq F(y), \forall y \in Y$. By (ii) and Definition 6, for each $N=\left\{y_{0}, \ldots, y_{n}\right\} \in\langle Y\rangle$, each $\left\{y_{i_{0}}, \ldots, y_{i_{n}}\right\} \in\langle N\rangle \quad$ and each $\quad z \in T\left(\varphi_{N}\left(\Delta_{k}\right)\right)$, 
$\min _{0 \leq j \leq k} g\left(y_{i_{j}}, z\right) \leq \lambda$. Hence there exists $r \in\{0, \ldots, k\}$ such that $g\left(y_{i_{r}, z}\right) \leq \lambda, \quad$ i.e., $z \in G\left(y_{i_{r}}\right) \subset \bigcup_{j=0}^{k} G\left(y_{i_{j}}\right) \subset \bigcup_{j=0}^{k} F\left(y_{i_{j}}\right) . \quad$ Since $z \in T\left(\psi_{N}\left(\Delta_{k}\right)\right)$ is arbitrary, we have

$$
T\left(\psi_{N}\left(\Delta_{k}\right) \subset \bigcup_{j=0}^{k} F\left(y_{i_{j}}\right) .\right.
$$

Hence, $F$ is a generalized $L-T-$ KKM mapping. The condition (iii) implies that $F$ is transfer compactly closed-valued. All conditions of Theorem 2 are satisfied. By Theorem 2 we have $\cap_{y \in Y} F(y) \neq \varnothing$. Then, there is $\hat{z} \in \cap_{y \in Y} F(y)$ such that

$$
f(y, \hat{z}) \leq \lambda, \forall y \in Y \text {. }
$$

Theorem 8 Let $(X, Y, \Psi),\left(Y, X, \Psi^{\prime}\right)$ be two FLS spaces and $Z$ be a topological space. Let $T: X \rightarrow 2^{Z}, \quad H: X \rightarrow 2^{Y}, \quad g: Y \times Z \rightarrow \mathrm{R} \cup\{ \pm \infty\}$. Assumption that

1. $\mathrm{g}$ is generalized 0 -L-quasiconcave wrt $\mathrm{T}$ in $\mathrm{y}$ and generalized 0 -L-quasiconvex wrt $\mathrm{H}$ in $\mathrm{z}$;

2. $\mathrm{g}$ is 0 -transfer compactly lower semicontinuous in $\mathrm{z}$ and 0 -transfer compactly upper semicontinuous in $\mathrm{y}$;

3. $T \in \mathrm{L}-\mathrm{KKM}(\mathrm{X}, \mathrm{Y}, \mathrm{Z})$; there is $S_{1}: Y \rightarrow 2^{X}$ such that $\mathrm{Y}$ is an L- $S_{1}$-subset of itself and $\overline{T\left(S_{1}(Y)\right)}$ is compact;

4. $H \in \mathrm{L}-\mathrm{KKM}(\mathrm{X}, \mathrm{Z}, \mathrm{Y})$; there is $S_{2}: Z \rightarrow 2^{X}$ such that $\mathrm{Z}$ is an L- $S_{2}$-subset of itself and $\overline{T\left(S_{2}(Z)\right)}$ is compact.

Then, $\mathrm{g}$ has a saddle point $(\hat{y}, \hat{z}) \in Y \times Z$, i.e., $g(y, \hat{z}) \leq g(\hat{y}, \hat{z}) \leq g(\hat{y}, z), \forall(y, z) \in Y \times Z$.

In particular, we have

$\inf _{z \in Z} \sup _{y \in Y} g(y, z)=\sup _{y \in Y} \inf _{z \in Z} g(y, z)=0$.

Proof. Applying Theorem 7 with $\lambda=0$ and $f \equiv g$, there exists a point $\hat{z} \in Z$ such that $g(y, \hat{z}) \leq 0$ for all $y \in Y$. Let $f(z, y)=-g(y, z)$ for all $(z, y) \in Z \times Y$. We apply Theorem 7 with $\lambda=0$ again, there is a point $\hat{y} \in Y$ such that $f(z, \hat{y}) \leq 0$ for all $z \in Z$. Then we have $g(y, \hat{z}) \leq 0 \leq g(\hat{y}, z), \forall(y, z) \in Y \times Z$. Thus, $g(\hat{y}, \hat{z})=0$ and

$g(y, \hat{z}) \leq g(\hat{y}, \hat{z}) \leq g(\hat{y}, z), \forall(y, z) \in Y \times Z$, which implies

$\inf _{z \in Z} \sup _{y \in Y} g(y, z) \leq g(\hat{y}, \hat{z}) \leq \sup _{y \in Y} \inf _{z \in Z} g(y, z)$

Since $\quad \inf _{z \in Z} \sup _{y \in Y} g(y, z) \geq \sup _{y \in Y} \inf f_{z \in Z} g(y, z) \quad$ is always hold, we get

$\inf _{z \in Z} \sup _{y \in Y} g(y, z)=\sup _{y \in Y} \inf f_{z \in Z} g(y, z)=0$.

W
Remark 9 Theorem 8 contains Theorem 4.2 of [25].

Theorem 9 Let $(X, Y, \Psi)$ be a FLS -space and $Z$ be a topological space. Let $T: X \rightarrow 2^{Z}, f, g: Y \times Z \rightarrow R \cup\{ \pm \infty\}$ and $\alpha, \beta \in R$ with $\alpha \leq \beta$ be such that

1. for each $(y, z) \in Y \times Z, \alpha \leq g(y, z) \leq \beta$ implies $\alpha \leq f(y, z) \leq \beta$;

2. $\mathrm{g}$ is generalized $\alpha-\beta$-L-quasiconcave wrt $\mathrm{T}$ in $\mathrm{y}$;

3. $\mathrm{f}$ is $\beta$-transfer compactly lower semicontinuous in $\mathrm{z}$ and $\alpha$-transfer compactly upper semicontinuous in $\mathrm{Z}$;

4. $T \in \mathrm{L}-\mathrm{KKM}(\mathrm{X}, \mathrm{Y}, \mathrm{Z})$; There is $S: Y \rightarrow 2^{X}$ such that $\mathrm{Y}$ is an L-S-subset of itself and $\overline{T(S(Y))}$ is compact.

Then, there exists a point $\hat{z} \in Z$ such that $\alpha \leq f(y, \hat{z}) \leq \beta, \forall y \in Y$.

Proof. We also define two mappings $F, G: Y \rightarrow 2^{Z}$ by

$F(y)=\{z \in Z: \alpha \leq f(y, z) \leq \beta\}$ and

$G(y)=\{z \in Z: \alpha \leq g(y, z) \leq \beta\}, \forall y \in Y$

Then, by (i), we have $G(y) \subseteq F(y)$ for all $y \in Y$. By (ii), for each $N=\left\{y_{0}, \ldots, y_{n}\right\} \in\langle Y\rangle$, each $\left\{y_{i_{0}}, \ldots, y_{i_{k}}\right\} \subset N$ and each $z \in T\left(\varphi_{N}\left(\Delta_{k}\right)\right)$, there is an $r \in\{0, \ldots, k\}$ satisfying $\alpha \leq g\left(y_{i_{r}}, z\right) \leq \beta$. It follows that $z \in\left\{z \in Z: \alpha \leq g\left(y_{i_{r}}, z\right) \leq \beta\right\}=G\left(y_{i_{r}}\right) \subset F\left(y_{i_{r}}\right) \subset \bigcup_{j=0}^{k} F\left(y_{i_{k}}\right)$.

Since $z \in T\left(\psi_{N}\left(\Delta_{k}\right)\right)$ is arbitrary, we have $T\left(\psi_{N}\left(\Delta_{k}\right)\right) \subset \bigcup_{j=0}^{k} F\left(y_{i j}\right)$. Hence $F$ is a $L-T-\mathrm{KKM}$ mapping.

We set

$A(y):=\{z \in Z: f(y, z) \leq \beta\}$

$B(y):=\{z \in Z: f(y, z) \geq \alpha\}$

Then one has $F(y)=A(y) \cap B(y)$. The condition (iii) implies that $A$ and $B$ are transfer compactly closed-valued. We need show that $F$ is transfer compactly closed-valued. For each compact subset $K$ of $Z$, by Lemma 1, we have

$\bigcap_{y \in Y}(A(y) \cap K)=\bigcap_{y \in Y}\left(\mathrm{c} l_{K} A(y) \cap K\right)$

and

$\bigcap_{y \in Y}(B(y) \cap K)=\bigcap_{y \in Y}\left(\mathrm{c} l_{K} B(y) \cap K\right)$.

It follows that 


$$
\bigcap_{y \in Y}([A(y) \cap B(y)] \cap K)=\bigcap_{y \in Y}\left(\left[\mathrm{cl} l_{K} A(y) \cap \mathrm{cl} l_{K} B(y)\right] \cap K\right) .
$$

On the other hand,

$$
\begin{aligned}
\bigcap_{y \in Y}([A(y) \cap B(y)] \cap K) & \subseteq \bigcap_{y \in Y}\left(\mathrm{cl}_{K}[A(y) \cap B(y)] \cap K\right) \\
& \subseteq \bigcap_{y \in Y}\left(\left[\mathrm{cl} l_{K} A(y) \cap \mathrm{cl} l_{K} B(y)\right] \cap K\right) .
\end{aligned}
$$

Therefore $F$ is transfer compactly closedvalued. Clearly, all conditions of Theorem 2 are satisfied. Applying theorem $2 \bigcap_{y \in Y} F(y) \neq \varnothing$. Taking $\hat{z} \in \bigcap_{y \in Y} F(y)$, we obtain $\hat{z} \in Z$ such that

$$
\alpha \leq f(y, \hat{z}) \leq \beta, \forall y \in Y \text {. }
$$

\section{REFERENCES}

[1] R.P. Agarwal, M. Balaj and D. O'Regan, "Common fixed point theorems and minimax inequalities in locally convex Hausdorff topological vector spaces," Appl. Anal., vol. 88, pp. 1691-1699, 2009.

[2] R.P. Agarwal, M. Balaj and O'Regan, "A Common fixed point theorem and applications," J. Optim. Theory Appl., vol 163, pp. 482-490, (2014).

[3] M. Balaj, "An intersection theorem with applications in minimax theory and equilibrium problem," J. Math. Anal. Appl., vol 336, pp. 363-371, 2007.

[4] T.H. Chang and C.L. Yen, "KKM property and Fixed point theorems," J. Math. Anal. Appl., vol 203, pp. 224-235, 1996.

[5] L. Deng and X. Xia, "Generalized -KKM type theorems in topological spaces with an application," J. Math. Anal. Appl., vol 285, pp. 679-690, 2003.

[6] X.P. Ding, "Generalized G-KKM type theorems in generalized convex spaces and their application," J. Math. Anal. Apll., vol. 229, pp. 21-37, 2002.

[7] X.P. Ding, "Generalized KKM type theorems in FC-spaces with applications (I)," J. Glob. Optim., vol. 36, pp. 581596, 2006.

[8] X.P. Ding, "Maximal elements of -majorized mappings in product FC-spaces and applications (I)," Nonlinear Anal., vol. 67, pp. 963-973, 2007.

[9] X.P. Ding, "New generalized R-KKM theorems in General Topological Space and Applications," Acta. Math. Sinica. English series, vol. 23, pp. 1969-1880, 2007.

[10] M. Fakhar and J. Zafanari, "Generalized R-KKM theorems and their application," Taiwanese. J. Math., vol 11, pp. 95$105,2007$.

[11] M. Fang and N.J. Huang, "Generalized -KKM type theorems in topological spaces with an application," $J$. Comput. Math. App., vol. 53, pp. 1896-1903, 2006.

[12] J. Huang, "The matching theorems and coincidence theorems for generalized -KKM mapping in topological spaces," J. Math. Anal. Appl., vol. 312, pp. 374-382, 2005.

[13] S.A. Khan, J. Iqbal and Y. Shehu, "Mixed quasi-variational inequalities involving error bounds," J. Inequal. Appl., pp. 2015-417 (2017).

[14] P.Q. Khanh, L.J. Lin, and V.S.T. Long, “On topological existence theorems and applications to optimization-related problems," Mathematical Methods of Operations Research, vol. 79, pp. 253-â€"272, 2014

[15] P.Q. Khanh and V.S.T. Long, "Invariant-point theorems and existence of solutions to optimization-related problems," J. Global Optim., vol. 58, pp. 545â€"-564, (2014.

[16] P.Q. Khanh and V.S.T. Long, "Weak Finite Intersection Characterizations of Existence in Optimization," Bull. Malays. Math. Sci. Soc., Onlinefirst.

[17] P.Q. Khanh,V.S.T. Long and N.H. Quan, "Continuous selections, collectively fixed points and weakly KnasterKuratowski-Mazukiewicz mappings in optimization," $J$. Optim. Theory Appl., vol. 151, pp. 552-572, 2011.

[18] P.Q. Khanh, N.H. Quan and Y.C. Yao, "Generalized KKM type theorems in GFC-spaces and applications," Nonlinear Anal., vol. 71, pp. 1227-1234, 2009.

[19] P.Q. Khanh and N.H. Quan, "Intersection theorems, coincidence theorems and maximal-element theorems in GFC-spaces," Optimization, vol. 59, pp. 115-124, 2010.

[20] P.Q. Khanh and N.H. Quan, "The solution existence of general inclusions using generalized KKM theorems with applications to minimax problems," J. Optim. Theory Appl., vol. 146, pp. 640-653, 2010.

[21] L.J. Lin, "A KKM type theorem and its applications," Bull. Austral. Math. Soc., vol. 59, pp. 481-493, 1999.

[22] L.J. Lin, "Variational relation problems and equivalent forms of generalized Fan-Browder fixed point theorem with applications to Stampacchia equilibrium problems," $J$. Global Optim., vol. 53, pp. 215-229, 2012.

[23] S. Park, "Comments on the KKM theory on -space," Pan. Am. Math. J., vol. 18, pp. 61-71, 2008.

[24] Salahuddin and R.U. Verma, "Generalized Set Valued Vector Equilibrium Problems," Pan. Am. Math. J., vol. 27, pp. 79-97, 2017.

[25] K.K. Tan, "G-KKM theorems, minimax inequalities and saddle points," Nonlinear Anal., vol. 30, pp. 4151-4160, 1997.

[26] R.U. Verma, "Role of generalized KKM type selections in a class of minimax inequalities," Appl. Math. Lett., vol. 12, pp. 71-74, 1999.

[27] R.U. Verma, "G-H-KKM type theorems and their applications to a new class of minimax inequalities," Comput. math. App., vol. 37, 45-48, 1999.

Ha Manh Linh was with the Department of Mathematics, Vietnam National UniversityHoChiMinh City, University of Information Technology, Thu Duc district, Saigon, Vietnam email:linhhm@uit.edu.vn. 


\title{
Các định lý loại Knaster-Kuratowski- Mazurkiewicz và các áp dụng cho các bất đẳng thức minimax
}

\author{
Hà Mạnh Linh
}

Tóm tắt - Các định lý loại KanasterKuratowski-Mazurkiewicz đóng một vai trò quan trọng trong các lĩnh vực giải tích phi tuyến, tối ưu và toán ứng dụng. Kể từ khi xuất hiện, nhiều nhà nghiên cứu đã nỗ lực phát triển các điều kiện đủ cho sự tồn tại các điểm giao (và các áp dụng của chúng) trong các không gian tổng quát như: Các không gian G-Lồi $[21,23]$, không gian L-lồi [12], và FC-không gian [8,9]

Các áp dụng của các định lý loại KanasterKuratowski-Mazurkiewicz, đặc biệt là nghiên cứu sự tôn tại cho các bất đẳng thức biến phân, các bài toán cân bằng và các bài toán tổng quát khác đã được thu được bởi nhiều tác giả, xem các bài báo gần đây $[1,2,3,8,18,24,26]$ và trong các tài liệu tham khảo của các bài báo này.

Trong bài báo này, chúng tôi đề xuất khái niệm ánh xạ L-T-KKM nhằm bao hàm các định nghĩa ánh xạ R-KKM [5], ánh xạ L-KKM [11], ánh xạ T-KKM ơ18,19], và các khái niệm đã có gần đây. Các định lý KKM suy rộng là được thiết lập để mở rộng các kết quả trước đó. Trong phần áp dụng, chúng tôi phát triển các định lý minimax ở dạng tổng quát. Các kết quả chúng tôi được chỉ ra là cải tiến hoặc chứa các kết quả khác như trường hợp đặc biệt.

Tù̀ khóa - Các ánh xạ L-T-KKM; Lồi suy rộng; Truyền compact nữa liên tục dưới, Các định lý minimax, Các điểm yên ngựa vô hạn. 